\title{
THRUST LAW EFFECTS ON THE LONG-PERIOD MODES OF AEROSPACE CRAFT
}

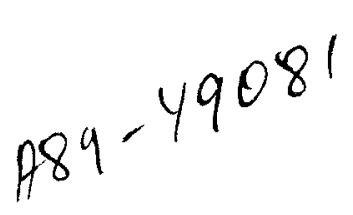

\author{
Nikos Markopoulos* and Kenneth D. Mease** \\ Mechanical and Aerospace Engineering Department \\ Princeton University, Princeton, New Jersey \\ and
}

Nguyen X. Vinh ${ }^{\dagger}$

Aerospace Engineering Department

University of Michigan, Ann Arbor, Michigan

\begin{abstract}
An analytical study is presented of the longitudinal long-period dynamics of an aerospace craft in a nearly circular orbit, with a thrust law depending arbitrarily on the speed and altitude. The effects of the thrust law on the longitudinal modes are explored. A plane of engine possibilities is first defined, with points corresponding to propulsion systems having prescribed thrust slopes with respect to speed and altitude. The characteristic equation governing the purely translational modes is then examined, leading to a fairly general stability picture in the plane of engine possibilities. Approximate expressions for the characteristic roots and times follow quite naturally, by first identifying a small quantity in the coefficients of the characteristic equation, and then expanding in a perturbation series about the origin of the plane of engine possibilities, for which the solution is always known. These expressions agree very well with the exact solutions over a wide range of altitudes and thrust laws. The period of the oscillatory translational mode (phugoid) is found to be independent to first order of the thrust law, generalizing results found by previous investigators for specific thrust laws. The results apply to the speed range from hypersonic to orbital.
\end{abstract}

\section{Nomenclature}

$\mathrm{x}, \mathrm{y}, \mathrm{z}=$ principal body axes with origin at the center of mass

$\mathrm{A}, \mathrm{B}, \mathrm{C}=$ principal $\mathrm{x}, \mathrm{y}, \mathrm{z}$ moments of inertia

$\mathrm{T}=$ thrust

$\mathrm{D}=\mathrm{drag}$

$\mathrm{X}=\mathrm{T}-\mathrm{D}$

$\delta_{\mathrm{T}} \quad=$ throttle setting

$\mathrm{u} \quad=$ flight speed

$\gamma \quad=$ flight path angle

$\mathrm{r} \quad=$ radius (altitude) of flight

* Graduate Research Assistant

** Assistant Professor, Senior Member AIAA

+ Professor

Copyright (C) American Institute of Aeronautics and Astronautics, Inc., 1989. All rights reserved.

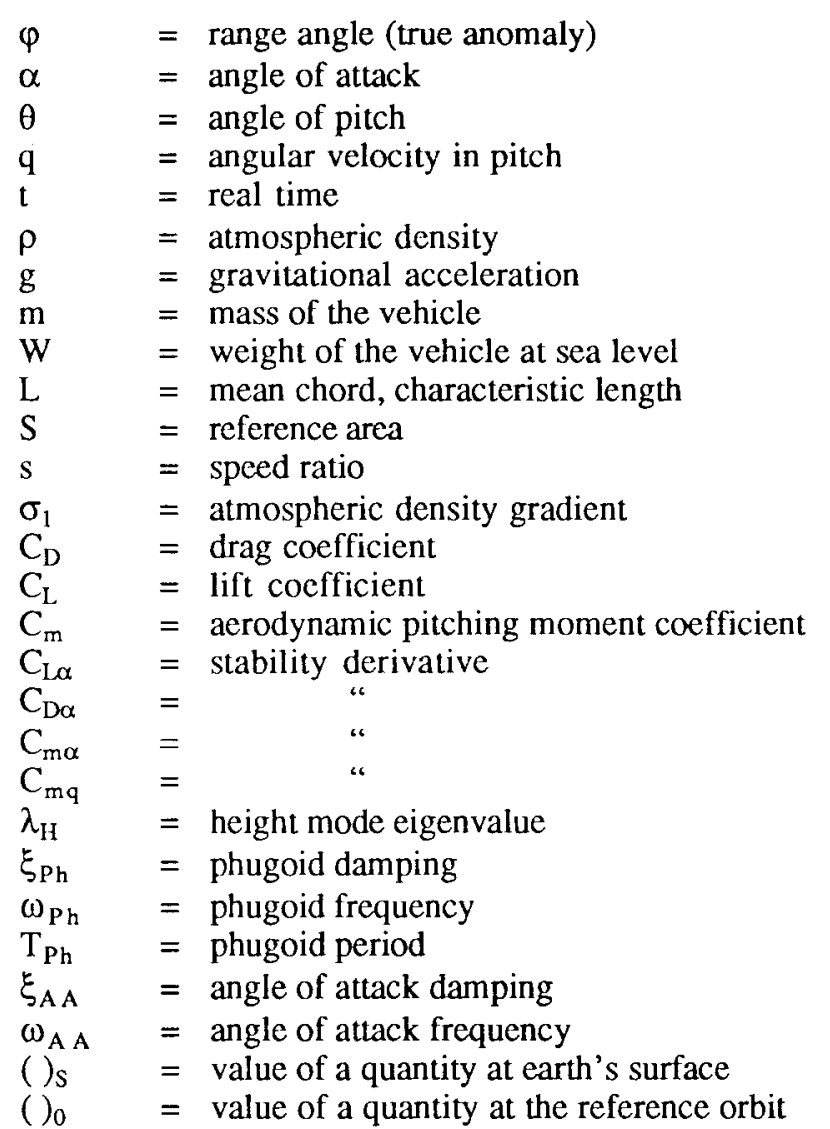

\section{Introduction}

There is a long legacy of studies ${ }^{1-12}$ of the nearsteady longitudinal dynamics of aircraft. For a rigid body model of an aircraft, there are six translational and six rotational state variables and there are six second order, or equivalently, twelve first order equations of motion. Steady flight is defined as flight with zero net forces and moments. Under the assumptions of a flat earth and uniform atmospheric density and gravity, a steady flight path is simply rectilinear motion at constant speed. If the density and gravity are modeled as functions of altitude, the steady path must be at constant altitude as well. A characteristic common to 
all steady flight is that thrust is used to cancel drag. For powered flight, the vehicle mass is changing, which adds an additional state variable and (first-order) equation of motion. For stability analysis, however, the mass change is generally neglected. Lanchester ${ }^{1}$ was the first to investigate analytically the dynamic stability of steady flight. Based on simplified, although nonlinear, equations, he identified a long-period trajectory oscillation, which he called the phugoid, characterized by an interchange between kinetic and potential energy with total energy almost constant.

Most subsequent stability studies focused on the eigenvalues and eigenvectors associated with the system of linear (time invariant) differential equations, obtained by linearization of the exact nonlinear equations about a steady flight condition. If the vehicle has a plane of symmetry, and certain other conditions, to be discussed later, are satisfied, the twelve linear equations decouple into two independent sets, associated with the longitudinal and the lateral-directional dynamics, respectively. The longitudinal dynamics involve four of the translational variables and two of the rotational variables. Generally, the models of the forces and moments do not involve one of the translational variables, namely the range or range angle, so that its dynamics are neglected in stability analyses. One is then left with a fifth-order linear system, whose stability is dictated by the five roots of the associated characteristic equation. Provided that none of the characteristic roots (eigenvalues) have zero real parts, the stability of the linear system characterizes the local stability of the nonlinear system.

In early studies of (linearized) longitudinal dynamics, which generally focused on subsonic flight, the atmospheric density and gravitational acceleration were assumed to be constant functions of altitude, the earth was assumed to be flat, and the flow about the vehicle was assumed to be incompressible. Under these assumptions, the altitude variation has no influence on the stability of an equilibrium solution for the remaining four longitudinal variables. An eigenvalue/eigenvector decomposition of the coefficient matrix for the fourth-order system generally uncovers two oscillatory modes: one lightly damped, longperiod, translational mode, corresponding to Lanchester's phugoid, and one heavily damped, shortperiod, rotational (angle of attack) mode. Neumark, ${ }^{4}$ concerned with longitudinal stability in high speed subsonic and supersonic flight, considered the fifth order system in order to treat compressibility and density gradient effects. For the fifth order system, there is a nonoscillatory (real) height or altitude mode (sometimes called the spiral mode) in addition to the two oscillatory modes.

In 1961, Etkin's considered the longitudinal dynamics of a lifting vehicle flying at near orbital speed. The gradients of density and gravity with altitude and the curvature of the steady reference flight path, due to the sphericity of the earth, were accounted for, in order that the resulting linearized equations of motion accurately predicted the near-steady, high-speed, high-altitude flight dynamics, including the limiting case of orbital motion in space. Note, however, that the aerodynamic coefficients were modeled as functions of only angle of attack. Etkin compiled the results of many numerical simulations and uncovered a number of interesting trends in the near-steady longitudinal dynamics. For example, the period of the phugoid mode approaches the orbital period as the altitude goes to infinity. Also, the period of the angle of attack mode increases with altitude, eventually becoming greater than the phugoid period at very high altitude. Laitone and $\mathrm{Chou}^{8}$ derived approximate analytical expressions for the period and damping of the phugoid and angle of attack oscillations that produced results in close agreement with those obtained numerically by Etkin. Vinh and Dobrzelecki ${ }^{10}$ improved the analytical approximations of Laitone and Chou using perturbation techniques, and explored both the linear coupling and the nonlinear effects near the altitude where the periods of the two modes become equal.

Given the current interest in hypersonic aerospace craft, it is appropriate to revisit the subject of nearsteady dynamics. As pointed out by Berry, 13 the thrust law for an aerospace craft may be more complicated than those considered by previous investigators. Etkin ${ }^{5}$ considered two thrust laws: one in which thrust was proportional to density, corresponding to what he termed a simple airbreathing engine, and one in which the thrust was constant, corresponding to a rocket engine. Laitone and Chou ${ }^{8}$ and Vinh and Dobrzelecki10 considered only constant thrust. Berry ${ }^{13}$ considered the thrust to be a function of Mach number, in which the slope of the thrust was positive over certain Mach number ranges and negative over others. Based on numerical simulations, he concluded that the thrust slope has a significant effect on the stability of the phugoid and height modes.

The objective of the work presented in this paper is to determine qualitatively the stability characteristics of the near-steady longitudinal dynamics of a vehicle with a propulsion system whose thrust law is a general function of the speed and altitude. Our formulation of the problem follows closely that of Vinh and Dobrzelecki10. Our approach is analytical in order to obtain results of a general nature that show explicitly the roles of the various parameters of the problem. We account for the density and gravity gradients with altitude and treat the earth as spherical. In this case the steady flight paths are segments of circles about an axis of the earth. However, the linearized longitudinal and lateral-directional dynamics are uncoupled, only if we consider segments of great circles. If the rotation of the earth is neglected, the great circles can be in any plane (passing through the center of the earth). We do not account for the earth's rotation, consistent with the 
studies mentioned above. This introduces some error and does not allow both an accurate modeling of aerodynamic forces and the correct limiting behavior in space; however the idealization does facilitate the development of analytical solutions. In obtaining quantitative results, we use the vehicle mass and aerodynamic data of Etkin ${ }^{5}$ (see Appendix B). This provides additional consistency and ease of comparison with the results of Refs. 5, 8, 10, and 13 .

\section{Equations of Motion and Equilibrium Solutions}

In terms of the state variables shown in Fig. 1, the equations of longitudinal motion for a lifting vehicle in the plane of a great circle of a nonrotating spherical earth are:

$$
\begin{aligned}
& \frac{d u}{d t}=\frac{T \cos \alpha}{m}-\frac{\rho S C_{D} u^{2}}{2 m}-g \sin \gamma \\
& \frac{d \gamma}{d t}=\frac{T \sin \alpha}{m u}+\frac{\rho S C_{L} u}{2 m}-\left(g-\frac{u^{2}}{r}\right)\left(\frac{\cos \gamma}{u}\right) \\
& \frac{d r}{d t}=u \sin \gamma \\
& \frac{d \varphi}{d t}=\frac{u \cos \gamma}{r} \\
& \frac{d \alpha}{d t}=q-\frac{T \sin \alpha}{m u}-\frac{\rho S C_{L} u}{2 m}+\frac{g \cos \gamma}{u} \\
& \frac{d q}{d t}=\frac{\rho S L C_{m} u^{2}}{2 B}-\left(\frac{3 g}{2 r}\right)\left(\frac{A-C}{B}\right) \sin 2(\gamma+\alpha)
\end{aligned}
$$

Under the assumptions of constant mass, spherical earth, and thrust vector aligned with the longitudinal $x$ axis of the vehicle, the first four equations govern the translational motion of the center of mass of the vehicle while the last two govern the rotational (pitching) motion about it. The fourth equation, governing the range, is uncoupled from the rest so it will not be considered in the subsequent analysis.

We consider a thrust law that is an arbitrary function of the throttle setting, speed, altitude, and angle of attack of the vehicle

$$
\mathrm{T} \equiv \mathrm{f}\left(\delta_{\mathrm{T}}, \mathrm{u}, \mathrm{r}, \alpha\right)
$$

and the following models for gravity and atmospheric density:

$$
g=g_{S}\left(\frac{r_{s}}{r}\right)^{2}
$$

$$
\rho \equiv \rho(r)
$$

We also assume that the lift and drag coefficients are functions of the angle of attack only, while the pitching moment coefficient depends on both the angle of attack and the angular velocity in pitch of the vehicle. The independence of the aerodynamic coefficients from the Mach number is a good approximation in the hypersonic continuum regime which is our primary interest.

\section{Equilibrium Solutions}

A steady flight solution, which will be denoted using a zero subscript, is an equilibrium solution for the fifth order system, given by Eqs. (1)-(3), (5), and (6), and is obtained by determining constant values of the states that satisfy the differential equations. To simplify the analysis, we assume that the steady flight angle of attack is:

$$
\alpha_{0}=0
$$

Clearly, the steady flight path must be circular. Once its constant radius $r_{0}$ is specified, the remaining steady states and controls are given explicitly by

$$
\begin{aligned}
& \gamma_{0}=0 \\
& \mathrm{q}_{0}=-\frac{\mathrm{u}_{0}}{\mathrm{r}_{0}} \\
& \mathrm{u}_{0}=\left[\frac{\mathrm{g}_{0} \mathrm{r}_{0}}{1+\left(\frac{\left.\rho_{0} \mathrm{Sr}_{0} \mathrm{C}_{\mathrm{L} 0}\right)}{2 \mathrm{~m}}\right)}\right]^{1 / 2} \\
& \mathrm{~T}_{0} \equiv \mathrm{f}\left(\delta_{\mathrm{T} 0}, \mathrm{u}_{0}, \mathrm{r}_{0}, 0\right)=\frac{\rho_{0} \mathrm{SC}_{\mathrm{D} 0} \mathrm{u}_{0}^{2}}{2} \\
& \mathrm{C}_{\mathrm{m} 0}=0
\end{aligned}
$$

where it is implicitly assumed that the propulsion system has enough power so that a solution for the nominal throttle setting in Eq. (14) exists:

\section{Nondimensional Equations of Motion}

In terms of the new variables

$$
\hat{\mathrm{u}}=\frac{\mathrm{u}}{\mathrm{u}_{0}}, \quad \hat{\mathrm{r}}=\frac{\mathrm{r}}{\mathrm{r}_{0}}, \quad \hat{\mathrm{q}}=\frac{\mathrm{L}}{2 \mathrm{u}_{0}} \mathrm{q}, \quad \hat{\mathrm{t}}=\frac{\mathrm{g}_{0}}{\mathrm{u}_{0}} \mathrm{t}
$$

and the definitions

$$
\hat{T}=\frac{T}{T_{0}}, \quad \hat{\rho}=\frac{\rho}{\rho_{0}}, \quad \mu=\frac{\rho_{0} S u_{0}^{2}}{2 m g_{0}}, \quad k_{0}=\frac{A-C}{B}
$$


$s=\frac{\mathrm{u}_{0}}{\sqrt{\mathrm{g}_{0} \mathrm{r}_{0}}}, \quad \eta=\frac{2 \mathrm{r}_{0}}{\mathrm{~L}}, \quad \mathrm{k}_{\mathrm{y}}^{2}=\frac{\mathrm{B}}{\mathrm{m}}, \quad \delta=\left(\frac{\mathrm{L}}{2 \mathrm{k}_{\mathrm{y}}}\right)^{2}$

Eqs. (1)-(3), (5), and (6) assume the nondimensional form

$$
\begin{aligned}
& \frac{d \hat{u}}{d \hat{t}}=\mu C_{D 0} \hat{T} \cos \alpha-\mu \hat{\rho} \hat{u}^{2} C_{D}-\frac{\sin \gamma}{\hat{r}^{2}} \\
& \frac{d \gamma}{d \hat{t}}=\frac{\mu C_{D 0} \hat{T} \sin \alpha}{\hat{u}}+\mu \hat{\rho} \hat{u} C_{L} \\
& \frac{d \hat{r}}{d \hat{t}}=s^{2} \hat{u} \sin \gamma \quad-\left(\frac{1}{\hat{r}^{2}}-\frac{s^{2} \hat{u}^{2}}{\hat{r}}\right)\left(\frac{\cos \gamma}{\hat{u}}\right) \\
& \frac{d \alpha}{d \hat{t}}=s^{2} \eta \hat{q}-\frac{\mu C_{D 0} \hat{T} \sin \alpha}{\hat{u}}-\mu \hat{\rho} \hat{u} C_{L}+\frac{\cos \gamma}{\hat{u}^{2}} \\
& \frac{d \hat{r}}{d \hat{t}}=2 \mu \delta \hat{\rho} \hat{u}^{2} C_{m}-\left(\frac{3 k_{0}}{2 \eta \hat{r}^{3}}\right) \sin 2(\gamma+\alpha)(20)
\end{aligned}
$$

\section{Linearized Equations of Motion}

For the purpose of studying the small motions about a reference circular orbit, we model the acrodynamic coefficients by

$$
\begin{aligned}
& C_{D}=C_{D 0}+C_{D \alpha} \alpha \\
& C_{L}=C_{L 0}+C_{L \alpha} \alpha \\
& C_{m}=C_{m \alpha} \alpha+C_{m q}\left(q-q_{0}\right)
\end{aligned}
$$

introduce the perturbation vector $\Delta \mathbf{Y}$

$$
\Delta Y=\left[\begin{array}{c}
\Delta \hat{\mathrm{u}} \\
\Delta \gamma \\
\Delta \hat{\mathrm{r}} \\
\Delta \alpha \\
\Delta \hat{\mathrm{q}}
\end{array}\right]=\left[\begin{array}{c}
\hat{\mathrm{u}}-1 \\
\gamma \\
\hat{\mathrm{r}}-1 \\
\alpha \\
\hat{\mathrm{q}}+\frac{1}{\eta}
\end{array}\right]
$$

and define the nondimensional quantities

$$
\begin{aligned}
& \mathrm{X}_{\mathrm{u}}=\left(\frac{\mathrm{u}_{0}}{\mathrm{~T}_{0}}\right)\left[\frac{\partial(\mathrm{T}-\mathrm{D})}{\partial \mathrm{u}}\right]_{0}=\left(\frac{\mathrm{u}_{0}}{\mathrm{~T}_{0}}\right)\left(\frac{\partial \mathrm{T}}{\partial \mathrm{u}}\right)_{0}-2 \\
& \mathrm{X}_{\mathrm{r}}=\left(\frac{\mathrm{r}_{0}}{\left|\sigma_{1}\right| \mathrm{T}_{0}}\right)\left[\frac{\partial(\mathrm{T}-\mathrm{D})}{\partial \mathrm{r}}\right]_{0}=\left(\frac{\mathrm{r}_{0}}{\left|\sigma_{1}\right| \mathrm{T}_{0}}\right)\left(\frac{\partial \mathrm{T}}{\partial \mathrm{r}}\right)_{0}+1 \\
& \mathrm{X}_{\alpha}=\left(\frac{1}{\mathrm{~m} \mathrm{~g}_{0}}\right)\left[\frac{\partial(\mathrm{T}-\mathrm{D})}{\partial \alpha}\right]_{0}=\mu\left[\left(\frac{\mathrm{C}_{\mathrm{D} 0}}{\mathrm{~T}_{0}}\right)\left(\frac{\partial \mathrm{T}}{\partial \alpha}\right)_{0}-\mathrm{C}_{\mathrm{D} \alpha}\right]
\end{aligned}
$$

with $\sigma_{1}$, the first order atmospheric density gradient, given as

$$
\sigma_{1}=\frac{r_{0}}{\rho_{0}}\left(\frac{\mathrm{d} \rho}{\mathrm{d} r}\right)_{0}
$$

Note that the three quantities introduced in Eqs. (25)(27) correspond to the nondimensional slopes of the difference between the thrust and the drag with respect to the speed, altitude, and angle of attack, respectively. Also, following Vinh and Dobrzelecki, ${ }^{10}$ we introduce the nondimensional quantity

$$
\omega^{2}=\left(1-s^{2}\right)\left(2-\sigma_{1} s^{2}\right)+s^{4}
$$

and the new time variable

$$
\tau=\omega \hat{\mathrm{t}}=\left(\frac{\omega \mathrm{g}_{0}}{\mathrm{u}_{0}}\right) \mathrm{t}
$$

Then, in terms of the perturbation vector, the equations of motion can be written as

$$
\frac{\mathrm{d}(\Delta \mathbf{Y})}{\mathrm{d} \tau}=\mathbf{f}(\Delta \mathbf{Y})=\mathbf{A} \Delta \mathbf{Y}+\mathbf{g}(\Delta \mathbf{Y})
$$

where, $\mathbf{A}$, the Jacobian of $\mathbf{f}$ evaluated at $\Delta \mathbf{Y}=\mathbf{0}$, is the constant matrix

$$
A=\left(\frac{1}{\omega}\right)\left[\begin{array}{ccccc}
\mu C_{D 0} X_{u}-1-\mu C_{D 0} \sigma_{1} X_{r} & X_{\alpha} & 0 \\
2 & 0 & A_{23} & A_{24} & 0 \\
0 & s^{2} & 0 & 0 & 0 \\
A_{41} & 0 & A_{43} & -A_{24} & s^{2} \eta \\
0 & -\frac{3 k_{0}}{\eta} & 0 & A_{S 4} 2 \mu \delta C_{m \hat{q}}
\end{array}\right]
$$

with $\mathrm{C}_{\mathrm{mq}}=\left(2 \mathrm{u}_{0} / \mathrm{L}\right) \mathrm{C}_{\mathrm{mq}}$, and 


$$
\begin{aligned}
& A_{23}=\left(1-s^{2}\right) \sigma_{1}+2-s^{2} \\
& A_{24}=\mu\left(C_{D 0}+C_{L \alpha}\right) \\
& A_{41}=s^{2}-2 \\
& A_{43}=\left(s^{2}-1\right) \sigma_{1}-2 \\
& A_{54}=2 \mu \delta C_{m \alpha}-\frac{3 k_{0}}{\eta}
\end{aligned}
$$

The function $\mathrm{g}$ specifies the higher order terms in the components of the perturbation vector.

For sufficiently small perturbations about the reference circular orbit, the purely dynamical (throtule setting fixed) motion of the vehicle is governed by the linear, time invariant equation

$$
\frac{\mathrm{d}(\Delta \mathrm{Y})}{\mathrm{d} \tau}=\mathbf{A} \Delta \mathbf{Y}
$$

More precisely, if the equilibrium solution is hyperbolic (i.e., none of the eigenvalues of $\mathbf{A}$ have zero real parts), then the eigenvalues and eigenvectors of $\mathbf{A}$ characterize the local behavior of the nonlinear system (30). 14

\section{Structure of the Linearized Dynamics}

In order to discuss the special structure of the Jacobian matrix A, that will be exploited subsequently, we write

$$
\mathbf{A}=\left[\begin{array}{ll}
\mathbf{A}_{\mathrm{TT}} & \mathbf{A}_{\mathrm{TR}} \\
\mathbf{A}_{\mathrm{RT}} & \mathbf{A}_{\mathrm{RR}}
\end{array}\right]
$$

where $\mathbf{A}_{\mathbf{T T}}$ is the $3 \times 3$ upper left block, associated with the translational variables and $\mathbf{A}_{\mathbf{R}}$ is the lower right block, associated with the rotational variables. The upper right block $\mathbf{A}_{\mathrm{TR}}$ accounts for the coupling from the rotational variables to the translational variables. The lower left block $\mathbf{A}_{\mathbf{R T}}$ accounts for the coupling from the translational variables to the rotational variables. The eigenvalues of $\mathbf{A}$ are the solutions of the equation

$$
\operatorname{det}(\mathbf{A}-\lambda \mathbf{I})=0
$$

In terms of the diagonal blocks of $\mathbf{A}$, as well as the individual elements of $\mathbf{A}$, denoted by $\mathrm{a}_{\mathrm{ij}}$, it can be shown that

$$
\begin{aligned}
& \operatorname{det}(\mathbf{A}-\lambda \mathbf{I})=\operatorname{det}\left(\mathbf{A}_{\mathbf{T T}}-\lambda \mathbf{I}_{\mathbf{3 \times 3}}\right) \operatorname{det}\left(\mathbf{A}_{\mathbf{R R}}-\lambda \mathbf{I}_{\mathbf{2 \times 2}}\right)+ \\
& +\mathbf{a}_{14}\left[-\mathrm{a}_{21}\left(\mathrm{a}_{33}-\lambda\right) \mathrm{a}_{45} \mathrm{a}_{52}+\mathrm{a}_{23} \mathbf{a}_{32} \mathbf{a}_{41}\left(\mathrm{a}_{55}-\lambda\right)\right.
\end{aligned}
$$

$$
\begin{aligned}
& \left.-\left(a_{22}-\lambda\right)\left(a_{33}-\lambda\right) a_{41}\left(a_{55}-\lambda\right)-a_{21} a_{32} a_{43}\left(a_{55}-\lambda\right)\right]+ \\
& +a_{24}\left[\left(a_{11}-\lambda\right)\left(a_{33}-\lambda\right) a_{45} a_{52}-a_{13} a_{32} a_{41}\left(a_{55}-\lambda\right)+\right. \\
& \left.+a_{12}\left(a_{33}-\lambda\right) a_{41}\left(a_{55}-\lambda\right)+\left(a_{11}-\lambda\right) a_{32} a_{43}\left(a_{55}-\lambda\right)\right]
\end{aligned}
$$

for the particular sparse structure shown in Eq. (31). Note that $a_{14}=X_{\alpha} / \omega, a_{24}=A_{24} / \omega$, and the other $a_{i j}$ can be identified using Eqs. (31)-(36).

Etkins found that, at least for the particular set of vehicle data he considered, the eigenvalues of the $\mathbf{A}$ matrix are well-approximated by the union of the eigenvalues of $\mathbf{A}_{\mathbf{T T}}$ and $\mathbf{A}_{\mathbf{R R}}$. Using the same vehicle mass and aerodynamic data as did Etkin, we have found this approximation to be a good one for thrust laws corresponding to a rocket, a turbojet, and a variety of other functions of altitude and speed. Figures illustrating these results will be presented later. These results imply that the latter two terms in Eq. (40) can be neglected. Note that the terms are both equal to zero if the A matrix is either upper or lower block triangular or block diagonal.

For physically reasonable parameter values, the matrix $\mathbf{A}$ has two complex conjugate pairs of eigenvalues and one real eigenvalue. Etkin ${ }^{5}$ found, from examining the corresponding eigenvectors, that, for most steady altitudes, the real mode and one of the complex modes involve only the translational variables, while the other complex mode involves only the rotational variables. This is the situation, familiar for low-speed flight, of the phugoid and angle of attack modes, with the addition of the height mode that arises from consideration of the density gradient and the spherical earth. An approximate block diagonal structure of the matrix $\mathbf{A}$ is indicated. The two complex modes are typically oscillatory (although not undamped). The frequencics of the two oscillations approach each other as the steady flight altitude increases. In the altitude region in which the frequencies are close, the modes are no longer separated between the translational and rotational variables. ${ }^{5}$ In this altitude region, the eigenvalues of $\mathbf{A}$ are still well-approximated by the union of the eigenvalues of $\mathbf{A}_{\mathbf{T T}}$ and $\mathbf{A}_{\mathbf{R R}}$, but there is a one-way coupling from translational to rotational variables. ${ }^{5}$ This is consistent with an approximately lower block triangular structure for the matrix A. (We note that one might argue the separate analysis of translational and rotational dynamics on the basis of a time scale separation. This is appropriate at low altitude where the angle of attack oscillation is well-damped or with a tightly controlled angle of attack. It is not appropriate for the high altitude, uncontrolled case considered here.) 
If the structure of $\mathbf{A}$ is lower block triangular, the solution $\Delta \mathbf{Y}=\left[\Delta \mathbf{Y}_{\mathbf{T}}, \Delta \mathbf{Y}_{\mathbf{R}}\right]^{\mathrm{T}}$ to Eq. (37) is of the form:

$$
\begin{aligned}
\Delta Y_{T}(t) & =e^{A_{T T}\left(t-t_{0}\right)} \Delta Y_{T}\left(t_{0}\right) \\
\Delta Y_{R}(t) & =e^{A_{R R}\left(t-t_{0}\right)} \Delta Y_{R}\left(t_{0}\right)+ \\
& +\int_{t_{0}}^{t} e^{A_{R R}(t-\zeta)} A_{R T} \Delta Y_{T}(\zeta) d \zeta
\end{aligned}
$$

Thus, the translational motion drives the rotational motion, but not vice versa. In the altitude region in which the two oscillatory modes have similar frequencies, there is a resonance effect, provided that the damping of the angle of attack mode is small, which it is. Vinh and Dobrzelecki ${ }^{10}$ have considered the linear coupling and nonlinear effects in this region.

We will have more to say about the near resonance behavior later. In the next two sections, we consider the qualitative behavior of the eigenvalues of $A_{\text {TT }}$ as functions of the steady altitude and the thrust law. Since our primary concern is the effect of the thrust characteristics of airbreathing engines on the translational dynamics, the relevant steady flight altitudes are well below the resonance altitude $(\sim 146$ $\mathrm{km}$ for Etkin's vehicle data ${ }^{5}$ ). Thus, the eigenvalues of $A_{\text {TT }}$ characterize the stability of the height and phugoid modes. We focus on these modes, since the stability of the rotational mode is independent of the thrust law5.

\section{Stability of Height and Phugoid Modes}

The characteristic equation associated with the submatrix $\mathbf{A}_{\text {TT }}$ is:

$$
\lambda^{3}+a_{2} \lambda^{2}+\lambda+a_{0}=0
$$

with

$$
\begin{aligned}
& a_{2}=-K \omega^{2} X_{u} \\
& a_{0}=K\left[2 s^{2} \sigma_{1} X_{r}-\left(\omega^{2}-2\right) X_{u}\right]
\end{aligned}
$$

and

$$
K=\frac{\mu C_{D 0}}{\omega^{3}}
$$

For a given vehicle the quantities $\omega^{2}$ and s depend solely on altitude. The speed ratio $s$ starts out as a small positive quantity for low altitude, and monotonically increases, approaching unity asymptotically with increasing altitude; $\omega^{2}$ remains always greater than unity, reaching a maximum value at some altitude and decreasing towards unity beyond this altitude. Figs. 2 and 3 show these variations for the vehicle considered by Etkin.

It is clear that the stability of the phugoid and height modes depends on the quantities (stability derivatives) $X_{u}$ and $X_{r}$, defined by Eqs. (25) and (26), which in turn depend on the thrust law. For a rocket engine, the thrust is approximately constant; for a turbojet, the thrust is approximately proportional to the density. Accordingly, we have

$$
\begin{array}{ll}
X_{u}=-2, X_{r}=1 & \text { (rocket) } \\
X_{u}=-2, X_{r}=0 & \text { (turbojet) }
\end{array}
$$

These two cases correspond to the two thrust laws considered by Etkin. Since it is not clear what thrust laws will be appropriate or desirable for aerospace craft, we would like to characterize the stability of the height and phugoid modes for a wider range of engine possibilities. It turns out that we can do so for the entire $\left(X_{u}, X_{r}\right)$ plane. The rocket and turbojet cases are two points in this plane of engine possibilities. Clearly, not all points in this plane correspond to realizable engines, but it is as easy to characterize stability on the entire plane, as it is on some subset of it.

The first step in our stability analysis is to determine, for each point in the plane of engine possibilities, the number of eigenvalues in the right half of the complex plane. This is accomplished by applying Routh's stability criterion to the coefficients of the characteristic equation (43). ${ }^{18}$ We find that the plane of engine possibilities can be separated into four distinct regions. For all the points in a given region, there are the same number of eigenvalues in the right-half plane. The four regions correspond respectively to the cases of $0,1,2$, and 3 right-half plane eigenvalues. Fig. 4 illustrates a typical case. The boundaries of the regions are composed of segments of two intersecting lines $A$ and $B$, whose slopes are always finite, such that

$$
\text { slope }(B)=-\frac{1-\frac{\omega^{2}}{2}}{s^{2} \sigma_{1}}<-\frac{1}{s^{2} \sigma_{1}}=\operatorname{slope}(A)
$$

The values of the slopes are determined only by the steady flight altitude, corresponding to $\mathrm{r}_{0}$. The atmospheric density gradient $\sigma_{1}$ is always negative, so the slope of line $\mathrm{A}$ is always positive. The slope of line $B$ is positive for $\omega^{2}<2$ and negative for $\omega^{2}>2$. As shown in Fig. 3, $\omega^{2}$ is greater than two up to a critical altitude $(\sim 100 \mathrm{~km}$ for Etkin's vehicle). Above this critical altitude $\omega^{2}$ is less than two. Fig. 5 shows the behavior of the slopes as a function of altitude for Etkin's vehicle.

The second step of our stability analysis is to determine the eigenvalue types in each of the four regions. Examination of the discriminant associated 
with the characteristic equation (43) supplies this information. The discriminant can be put into the form: 17

$$
\mathrm{D}_{3}=\mathrm{PX}_{\mathrm{r}}^{2}+2 \mathrm{QX}_{\mathrm{r}}+\mathrm{R}
$$

with

$$
\begin{aligned}
& P=K^{2} s^{4} \sigma_{1}^{2} \\
& Q=\left(\frac{\omega^{2}}{3}+\frac{K^{2} \omega^{6} X_{u}^{2}}{27}-1\right) K^{2} s^{2} \sigma_{1} X_{u} \\
& R=\left(\frac{\omega^{2}-2}{27}\right) K^{4} \omega^{6} X_{u}^{4}+\left(\frac{2 \omega^{4}}{27}-\frac{2 \omega^{2}}{3}+1\right) K^{2} X_{u}^{2}+\frac{1}{27}
\end{aligned}
$$

At the points in the $\left(X_{u}, X_{r}\right)$ plane where $D_{3}$ is positive, there are one real and two complex conjugate eigenvalues. At the points where $D_{3}$ is negative, there are three real eigenvalues. The curves along which the equality

$$
X_{I}=-\frac{Q}{P} \pm \frac{\sqrt{Q^{2}-P R}}{P}
$$

is satisfied, separate the two regions. The quantity

$$
\Delta \equiv \mathrm{Q}^{2}-\mathrm{PR}
$$

can be written in the simple form:

$$
\Delta=\left(\frac{K^{2} s^{4} \sigma_{1}^{2}}{729}\right)\left[\left(K \omega^{2} X_{u}\right)^{2}-3\right]^{3}
$$

For negative $\Delta$ however, (52) is never satisfied, and the sign of $D_{3}$ in (48) is the same as the sign of $P$, that is, positive. It follows that, for

$$
\left|X_{u}\right|<\frac{\sqrt{3}}{K \omega^{2}}=\sqrt{3} \omega\left[\frac{2 m}{\rho_{0} S C_{D 0} r_{0}}+\frac{C_{L 0}}{C_{D 0}}\right]
$$

the characteristic equation is assured to have one real and two complex conjugate roots. The right hand side of this last inequality increases very fast with altitude, due to its inverse dependence on atmospheric density. A lower bound on it can be estimated at zero altitude as

$$
\sqrt{3}\left(\frac{C_{L 0}}{C_{D 0}}\right) \approx \text { order of } 10
$$

For the vehicle considered by Etkin, this right hand side of the inequality (55) starts out as 11 at $0 \mathrm{~km}$, goes to 190 at $50 \mathrm{~km}$, and rises to 17500 at $100 \mathrm{~km}$. On the other hand, magnitudes of the nondimensional slope $X_{v}$ that exceed 10 are unlikely, because they correspond to extremely sharp variations of the thrust with speed. Therefore, in practice, inequality (55) is always satisfied and the appearance of an oscillatory translational mode (phugoid) for the vehicle is always expected.

Fig. 6 shows the typical stability picture, for the plane of engine possibilities, that emerges from the above analysis. Quantitative information for the height and phugoid modes, based on Eq. (43), is given in Figs. (7)-(9), for Etkin's vehicle, at a steady flight altitude of $50 \mathrm{~km}$.

\section{Approximate Expressions for Eigenvalues}

In order to show explicitly the dependence of the eigenvalues associated with the height and phugoid modes on the parameters $\mathrm{K}, \mathrm{s}, \omega, \sigma_{1}, \mathrm{X}_{\mathrm{u}}$, and $\mathrm{X}_{\mathrm{r}}$, we shall use approximation techniques to derive simple analytical expressions. For a given steady flight altitude and for a given vehicle with unspecified propulsion system the roots of the characteristic equation (43) become functions of the generalized slopes $\mathrm{X}_{\mathrm{u}}$ and $\mathrm{X}_{\mathrm{r}}$ only. Furthermore, for $\mathrm{X}_{\mathrm{u}}=0, \mathrm{X}_{\mathrm{r}}=0$, the exact solutions of the characteristic equation are:

$$
\text { height mode: } \quad \lambda_{\mathrm{H}}=0
$$

$$
\text { phugoid: } \quad \lambda_{\mathrm{Ph}}=\xi_{\mathrm{Ph}}+\mathrm{i} \omega_{\mathrm{Ph}}=0+\mathrm{i} 1
$$

Since we know the solution at the point $(0,0)$, we can obtain the solution at any other point $\left(X_{u}, X_{r}\right)$ by expanding in a Taylor series, provided the eigenvalues are analytic functions of these stability derivatives. To this end, we differentiate repeatedly the characteristic equation and calculate all the necessary partial derivatives at the point $(0,0)$. The result, to first order in $X_{u}, X_{r}$, is:

$$
\begin{aligned}
& \lambda_{\mathrm{H}} \approx \mathrm{K}\left[\left(\omega^{2}-2\right) \mathrm{X}_{\mathrm{u}}-2 \mathrm{~s}^{2} \sigma_{1} \mathrm{X}_{\mathrm{r}}\right] \\
& \xi_{\mathrm{Ph}} \approx \mathrm{K}\left[\mathrm{X}_{\mathrm{u}}+\mathrm{s}^{2} \sigma_{1} \mathrm{X}_{\mathrm{r}}\right] \\
& \omega_{\mathrm{Ph}} \approx 1
\end{aligned}
$$

or, to second order in $X_{u}, X_{r}$ for $\omega_{P h}$

$$
\begin{aligned}
\omega_{\mathrm{Ph}_{\mathrm{h}}} \approx 1+\mathrm{K}^{2}[(3- & \left.2 \omega^{2}\right) \mathrm{X}_{\mathrm{u}}^{2}+3 \mathrm{~s}^{4} \sigma_{1}^{2} \mathrm{X}_{\mathrm{r}}^{2} \\
& \left.+2\left(3-\omega^{2}\right) \mathrm{s}^{2} \sigma_{1} \mathrm{X}_{\mathrm{u}} \mathrm{X}_{\mathrm{r}}\right]
\end{aligned}
$$

How useful the above expressions are depends of course on how well the first order terms approximate the exact roots. One can also view the eigenvalue problem as a regular perturbation problem in the parameter K. Expansion in a perturbation series in 
powers of $\mathrm{K}$ for the eigenvalues can be shown to lead precisely to the same result, expressed by Eqs. (59)(62). Since the slopes $X_{u}, X_{r}$ can be expected to be of order unity, and $\mathrm{K}$, being proportional to density, is small over a wide altitude range, as shown in Fig. 10, the approximation should be quite accurate.

Using Eqs. (59)-(61), approximate expressions for the characteristic times can now be derived. The times to double/half for the height mode and the phugoid amplitude are approximated, in real time, as

$$
\begin{aligned}
& \Delta t_{H} \approx \frac{2(\ln 2) m \omega^{2}}{\rho_{0} S u_{0} C_{D o}\left[\left(\omega^{2}-2\right) X_{u}-2 s^{2} \sigma_{1} X_{r}\right]} \\
& \Delta t_{P h} \approx \frac{2(\ln 2) m \omega^{2}}{\rho_{0} S u_{0} C_{D o}\left[X_{u}+s^{2} \sigma_{1} X_{r}\right]}
\end{aligned}
$$

with $\Delta \mathrm{t}$ denoting a time to double if $\Delta \triangleright 0$, and $-\Delta \mathrm{t}$ a time to half if $\Delta \mathbf{t}<0$. Similarly, to first order in $K$ the phugoid period in real time is:

$$
\mathrm{T}_{\mathrm{Ph}} \approx \frac{2 \pi \mathrm{u}_{0}}{\omega \mathrm{g}_{0}}
$$

This result, identical to that derived by Laitone and $\mathrm{Chou}^{8}$ for a rocket, indicates that the phugoid period is independent of the propulsion system of the vehicle. In Fig. 9 we have already presented some evidence of this. By examining the quadratic form in Eq. (62), it can be shown that the point $\left(X_{u}, X_{r}\right)=(0,0)$ of the solution surface of $T_{P h}$ is saddle-point-like (see Fig. 9) at all altitudes of interest. Under the flat earth and uniform gravity assumptions, the expression for the phugoid period reduces to that of Scheubel. ${ }^{3}$ If, in addition, the density gradient is ignored, the classical result of Lanchester ${ }^{1}$ is obtained, namely, $T=\sqrt{2} \pi \mathrm{u}_{0} / \mathrm{g}_{0}$. Note that by phugoid period we mean the time interval between positive zero crossings, since unless undamped, the phugoid is not periodic.

\section{Numerical Validation}

In order to test the validity of approximating three of the eigenvalues of the $5 \times 5$ matrix $\mathbf{A}$ by the eigenvalues of the $3 \times 3$ matrix $\mathbf{A}_{\mathbf{T T}}$ and the validity of the further approximation of the eigenvalues of $\mathbf{A}_{\text {TT }}$ using Eqs. (59)-(61) and (65), we have carried out quantitative comparisons, using Etkin's vehicle data, for many points in the $\left(X_{u}, X_{r}\right)$ plane and for many different altitudes. Of the five eigenvalues obtained for $A$, we select the real one and the complex pair with the smallest (positive) imaginary part for altitudes below resonance and with the largest (positive) imaginary part for altitudes above resonance. We have found that the selected eigenvalues of $\mathbf{A}$ are uniformly wellapproximated by the eigenvalues of $\mathbf{A}_{\text {TT }}$. Only near the resonance altitude $(\sim 146 \mathrm{~km}$ for Etkin's vehicle) is there some noticeable error. (We will return to this point later.) The simple analytical expressions have also been found to be quite accurate. Figs. 11-13 show the comparisons for the turbojet case.

\section{Interpretation of Stability Results}

We shall now discuss our results relative to those of Etkin $^{5}$ and Berry. ${ }^{13}$ Etkin concluded that the height mode is unstable for the rocket and asymptotically stable for the turbojet below a certain critical altitude $(\sim 100 \mathrm{~km}$ for his data). Recalling that for a rocket we have $X_{u}=-2$ and $X_{r}=1$, and for a turbojet $X_{u}=-2$ and $X_{r}=0$, Eq. (59) confirms these results. For a rocket, the destabilizing term involving $X_{r}$ always dominates the stabilizing term involving $X_{u}$, based on the behaviors of $\omega^{2}$ and $s$ shown in Figs. 2 and 3 and the fact that $\sigma_{1}$ is of the order of -600 . For a turbojet, only the $X_{u}$ term is nonzero; below the critical altitude, $\omega^{2}>2$ and the height mode is asymptotically stable. Regarding the phugoid mode, Etkin concluded that a rocket has good damping, while a turbojet has poor damping. The implications of Eq. (60) are consistent with this conclusion. The first term on the right-hand-side of Eq. (60) is the same for both rocket and turbojet; although it is stabilizing, it is extremely small, due to the smallness of $\mathrm{K}$. Thus, a turbojet has poor damping, since the second term is zero in this case. On the other hand, the second term is nonzero for the rocket, has a stabilizing effect, and is a couple of orders of magnitude larger than the first term. The result is good phugoid damping for a rocket. Finally, Etkin concluded that the thrust law has little effect on the phugoid frequency. Eq. (62) shows that the stability derivatives $X_{u}$ and $X_{r}$ contribute only second-order corrections to the phugoid frequency. Therefore, for the two points in the plane of engine possibilities, corresponding to a rocket and a turbojet, our results are consistent with those of Etkin.

Berry ${ }^{13}$ used Etkin's vehicle mass and aerodynamic data, ${ }^{5}$ but considered thrust as a function of Mach number. He concluded, based on numerical simulations, that a (locally) positive thrust versus Mach number slope produces an unstable height mode and an unstable phugoid; while a negative slope produces a stable height mode and a stable phugoid. Fig. 6 and Eqs. (59) and (60) show that the behavior is more complicated than this. The (local) slope of the thrust versus Mach number curve does not in itself determine the stability of the modes.

Based on our analysis, we state the following general conclusions:

- A positive value of $X_{u}$ results in at least one unstable translational mode. The value of $\mathrm{X}_{\mathrm{r}}$ determines which mode(s) are unstable. Conversely, a negative value of $X_{u}$ results in at least one stable translational mode. The value of $X_{\mathrm{r}}$ determines which mode(s) are stable. (See Fig. 6.) Note that it is the variation of thrust 
minus drag with speed, rather than the variation of thrust alone with speed, that is an important indicator of stability. Stengel ${ }^{11}$ noted this in his work.

- Increasing the value of $X_{r}$ has a destabilizing effect on the height mode and a stabilizing effect on the phugoid. This is seen in Fig. 6 and in Eqs. (59) and (60).

- The region of the plane of engine possibilities in which both translational modes are stable diminishes as the steady flight altitude increases, as shown by Figs. 5 and 6.

- The phugoid period is independent of the thrust law to first order in the parameter $\mathrm{K}$.

\section{Behavior at Extremely High Altitude}

As the altitude increases, airbreathing engines cease to be viable sources of thrust for cruising flight. However, there are several interesting features of the near-steady, longitudinal dynamics at extremely high altitude worth mentioning. In order to discuss these features, we need to consider the angle of attack oscillatory mode. We shall calculate the complex pair of eigenvalues associated with this mode from the $2 \times 2$ matrix $\mathbf{A}_{\mathbf{R R}}$. Comparison of these eigenvalues with the corresponding eigenvalues of the $5 \times 5$ matrix A shows that the approximation is a good one, except near the resonance altitude.

The damping and frequency (assuming that the term under the radical is negative) associated with the eigenvalues of $\mathbf{A}_{\mathbf{R R}}$ are respectively:

$$
\begin{aligned}
& \xi_{\mathrm{AA}}=\left(\frac{\mu}{2 \omega}\right)\left(2 \delta \mathrm{C}_{\mathrm{mA}}-\mathrm{C}_{\mathrm{D} 0}-\mathrm{C}_{\mathrm{L} \alpha}\right) \\
& \omega_{\mathrm{AA}}=\left(\frac{1}{2 \omega}\right) \sqrt{12 \mathrm{~s}^{2} \mathrm{k}_{0}-1_{1} \mu-1_{2} \mu^{2}}
\end{aligned}
$$

with

$$
\begin{aligned}
& \mathrm{l}_{1}=8 \delta \mathrm{s}^{2} \eta \mathrm{C}_{\mathrm{m} \alpha} \\
& \mathrm{l}_{2}=\left(2 \delta \mathrm{C}_{\mathrm{m} \hat{\mathrm{q}}}+\mathrm{C}_{\mathrm{D} 0}+\mathrm{C}_{\mathrm{L} \alpha}\right)^{2}
\end{aligned}
$$

We observe that they do not depend on the details of the thrust law or the density gradient. The damping is proportional to $\mu$. Since $\mu$ is proportional to density, the damping decreases as the altitude increases. Also, as the altitude increases and $\mu$ decreases, the period of the angle of attack oscillation increases, eventually surpassing the phugoid period. This behavior is illustrated in Fig. 14 in terms of the corresponding frequencies. Ekkin ${ }^{5}$ noted this behavior and stated that at the altitude at which the periods are equal, the angle of attack oscillation would be unstable. By equating the frequencies given by Eqs. (61) and (67), an approximate implicit expression for the resonance altitude can be obtained 10 . For Etkin's vehicle data, the resonance altitude is around $146 \mathrm{~km}$. Since the phugoid period was found to be independent of the thrust law to first order, the resonance altitude is also independent of the propulsion system of the vehicle.

An interesting feature of the linearized dynamics near this "resonance" altitude was discovered later by Vinh and Dobrzelecki10. We have confirmed their result. Fig. 15, which is a high-resolution view of the frequencies of the oscillatory modes of the $5 \times 5$ matrix $A$, shows that the frequencies, considered as functions of altitude, never cross as it appears in Fig. 14. The frequencies of the oscillatory modes of the submatrices $\mathbf{A}_{\mathbf{T T}}$ and $\mathbf{A}_{\mathbf{R R}}$ approximate well the frequencies of the oscillatory modes of $\mathbf{A}$ on either side of the resonance altitude, but the actual correspondence flip-flops. The eigenvectors associated with the lower frequency oscillatory mode of $A$ lie in the subspace of the translational variables for steady flight altitudes well below the resonance altitude. As the steady flight altitude increases and passes through the resonance region, the eigenvectors rotate into the subspace of the rotational variables. Around the resonance altitude, the lower frequency mode is not a purely translational (phugoid) mode or a purely rotational (angle of attack) mode. Reference to the phugoid as the long period mode is only appropriate below the resonance altitude. The description of the higher frequency mode is similar, except for the obvious differences.

As the steady flight altitude increases beyond that of resonance, the terms proportional to $\mu$ become negligible and the eigenvalues associated with the angle of attack oscillation are given by

$$
\lambda_{\mathrm{AA}} \approx \pm \sqrt{\frac{3(\mathrm{C}-\mathrm{A})}{\mathrm{B}}}
$$

where the limiting values $\omega^{2}=1$ and $s^{2}=1$ have been substituted. In words, we are left with the classical problem of the stability of the pitch dynamics of a vehicle in a circular orbit subject to a gravity-gradient torque. The relative sizes of the moments of inertia dictate the stability.

In the limit, as the steady altitude goes to infinity, the phugoid oscillation corresponds to a slightly elliptical orbit, and consequently, the phugoid period is the period of this elliptical orbit ${ }^{10}$.

\section{Conclusions}

Historically, much insight into the dynamics of aircraft has been achieved through the study of the modes of the linearized dynamics about a steady flight condition. With the current interest in hypersonic aerospace craft, it is appropriate to revisit this subject, in order to understand the implications of higher speeds, higher altitudes, etc. We have extended the results of several previous studies of the longitudinal dynamics by 
considering a wide range of engine possibilties. The engines are characterized by the local slope of the generated thrust with respect to altitude and speed. A general qualitative picture of the stability of the nearsteady dynamics has emerged from our analysis. The partial derivatives of the difference between thrust and drag with respect to speed and altitude play a key role in determining the stability of the translational dynamics. If thrust increases faster than drag as speed increases, at least one of the translational modes (height or phugoid) is unstable. Increasing the partial derivative of the difference between thrust and drag with respect to altitude has a destabilizing effect on the height mode and a stabilizing effect on the phugoid mode. On the other hand, the period of the phugoid mode is independent of the details of the thrust law to first order. We have only briefly discussed the angle of attack mode, since it is not influenced by the details of the thrust law. However, we have confirmed a previous result stating that the frequencies of the two oscillatory modes of the linearized dynamics never become equal as altitude increases, but only become nearly equal and then diverge. The altitude at which the frequencies are nearly equal is a (near) resonance altitude in that oscillations in the translational variables drive the angle of attack oscillation and cause instability.

Our results and those of previous investigators show that the eigenvalues of the $5 \times 5$ Jacobian matrix for the nonlinear dynamics are well-approximated by the union of the eigenvalues of the $3 \times 3$ submatrix associated with the translational variables and the eigenvalues of the $2 \times 2$ submatrix associated with the rotational variables. This property allows an analytical approach to stability analysis with consequent general results showing explicitly the role of the various parameters. For the sake of consistency, we have used the same mass and aerodynamic data used in previous studies to verify this property. It is not known whether this property is generic for aerospace craft. A strong variation of thrust with angle of attack could destroy this property.

As a final note, the validity of the modeling assumptions and the relevance of the local stabilty results to the actual flight of an aerospace craft have not been established.

\section{Appendix A}

All numerical computations are based on the U.S. Standard Atmosphere 1962.19 However, for ease of computation the approximate inverse polynomial representation of this atmosphere that appears in the U.S. Standard Atmosphere Supplements, $1966,{ }^{20}$ is assumed to be exact.

The inverse polynomial is of the form:

$$
-\rho / \rho_{s}=1 /\left[A_{0}+A_{1} h+\ldots \ldots+A_{11} h^{11}\right]^{4}
$$

where, $\mathrm{h}$ is the geometric altitude above the standard geoid (6378.17 $\mathrm{km}$ radius) in kilometers. The polynomial approximation is valid for the altitude range $0-200 \mathrm{~km}$. Compared to the Standard Atmosphere, the approximation differs by less then $5 \%$ in this altitude range.

\section{Appendix B}

The characteristics of the vehicle geometry and aerodynamic stability derivatives used in all numerical calculations are those used by Etkin ${ }^{5}$ and are typical for a slender body, a cone or wedge of $3^{\circ}$ semiangle. The values can be derived from the simple Newtonian impact theory for moderate angles of attack. They are tabulated below.

Table B-1 Geometric Vehicle Parameters

$$
\begin{array}{rr}
k_{y}=7.62 m & L=15.24 m \\
k_{0}=-0.94 & W / S=146.6 \mathrm{kp} / \mathrm{m}^{2} \text { (sea level) }
\end{array}
$$

Table B-2 Aerodynamic Stability Derivatives

$$
\begin{aligned}
C_{L 0} & =0.05 & C_{D 0}=0.0133 & \left(2 \mathrm{u}_{0} / \mathrm{L}\right) \mathrm{C}_{\mathrm{mq}}=-0.028 \\
\mathrm{C}_{\mathrm{I} \alpha} & =0.329 & \mathrm{C}_{\mathrm{D} \alpha}=0.15 & \mathrm{C}_{\mathrm{m} \alpha}=-0.0548
\end{aligned}
$$

\section{Acknowledgment}

The authors thank Professors H. C. Curtiss, Jr. and R. F. Stengel for helpful discussions. This research was supported in part by the NASA Langley Research Center under Grant No. NAG1-907.

\section{References}

1 Lanchester, F. W., Aerodynamics, Arnold Constable, London, 1907.

2 Bryan, G. H., Stability in Aviation, Macmillan Co., London, 1911.

${ }^{3}$ Scheubel, F. N., "The Effect of Density Gradient on the Longitudinal Motion of an Aircraft," Luftfahrtforschung, Vol. 19, No. 4, 1942, pp. 132-136; R.T.P. Translation 1739.

${ }^{4}$ Neumark, S., "Longitudinal Stability, Speed and Height," Aircraft Engineering, Vol. 22, Nov. 1950, London, pp. 323-334.

5 Etkin, B., "Longitudinal Dynamics of a Lifting Vehicle in Orbital Flight," J. Aerospace Sci., Vol. 28, Oct. 1961, pp. 779-788.

6 Rangi, R. S., "Non-Linear Effects in the Longitudinal Dynamics of a Lifting Vehicle in Orbital Flight," Univ. of Toronto, UTIA TN 40, Oct. 1960. 
7 Porter, R. F., "The Linearized Long-Period Longitudinal Modes of Aerospace Vehicles in Equilibrium Flight," USAF, Flight Test Center, Edwards AFB, AFFTC-TN-61-2, Jan. 1961.

8 Laitone, E. V. and Chou, Y. S., "Phugoid Oscillations at Hypersonic Speeds," AIAA Journal, Vol. 3, No. 4, April 1965, pp. 732-735.

${ }^{9}$ Larrabee, E. E., "Acrodynamic Penetration and Radius as Unifying Concepts in Flight Mechanics," Journal of Aircraft, Vol. 4, No. 1, Jan.-Feb. 1967, pp. 2835.

10 Vinh, N. X. and Dobrzelecki, A. J., "Non-Linear Longitudinal Dynamics of an Orbital Lifting Vehicle," NASA CR-1449, 1969.

11 Stengel, R. F., "Altitude Stability in Supersonic Cruising Flight," Journal of Aircraft, Vol. 7, No. 9, Sept.-Oct. 1970, pp. 464-473.

12 Sachs, G., "The Effects of Pitching-Moments on Phugoid and Height Mode in Supersonic Flight," Journal of Aircraft, Vol. 9, No. 3, March 1972, pp. 252254.

13 Berry, D. T., "Longitudinal Long-Period Dynamics of Aerospace Craft," Paper No. 88-4358, AIAA Atmospheric Flight Mechanics Conference, Minneapolis, Minnesota, August 15-17, 1988.

14 Guckenheimer, J. and Holmes, P., Nonlinear Oscillations, Dynamical Systems, and Bifurcations of Vector Fields, Springer-Verlag, New York, 1983.

15 Etkin, B., Dynamics of Atmospheric Flight, John Wiley \& Sons, New York, 1972.

16 McRuer, D., Ashkenas, I., and Graham, D., Aircraft Dynamics and Automatic Control, Princeton Univ. Press, Princeton N.J., 1973.

17 Spiegel, M. R., Mathematical Handbook of Formulas and Tables, Schaum's Outline Series, McGraw-Hill, New York, 1968.

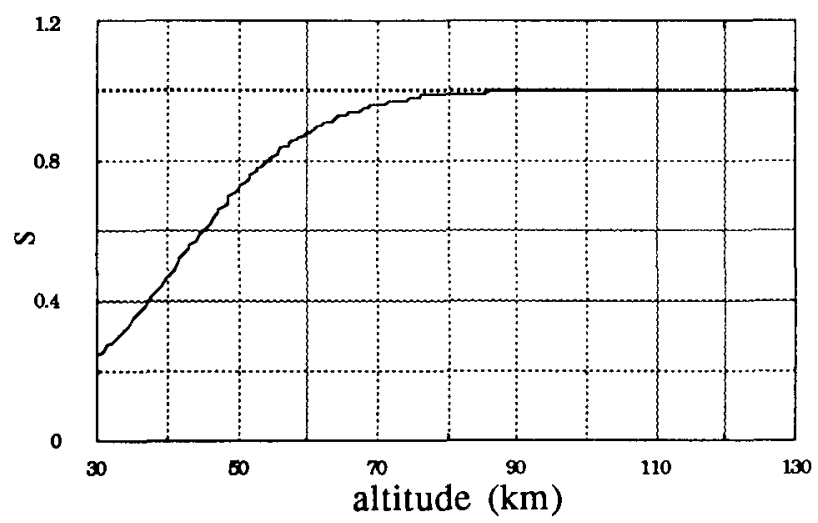

Fig. 2 Variation of $s$ with altitude for Etkin's vehicle
18 D'Azzo, J. J., and Houpis, C. H., Linear Control System Analysis and Design, McGraw-Hill, New York, 1981.

19 U.S. Standard Atmosphere, 1962, prepared under sponsorship of ESSA, NASA, and USAF.

20 U.S. Standard Atmosphere Supplements, 1966, prepared under sponsorship of NASA, USAF, and U.S. Weather Bureau.

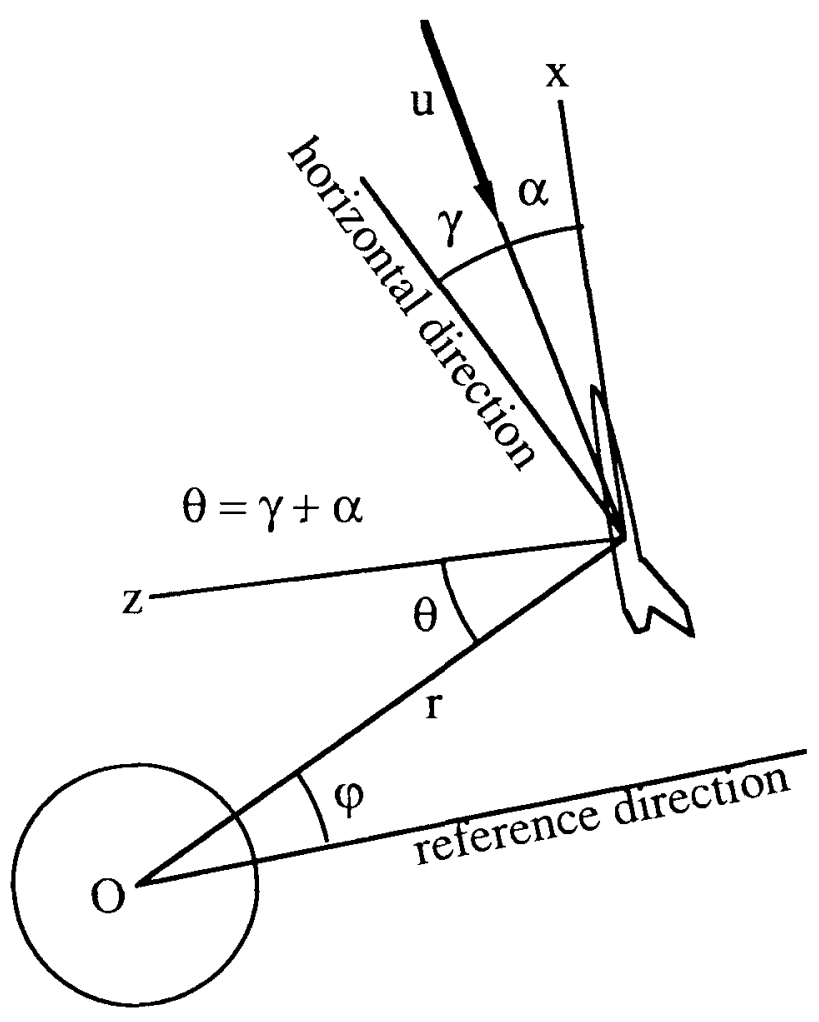

Fig. 1 State variables and axes system

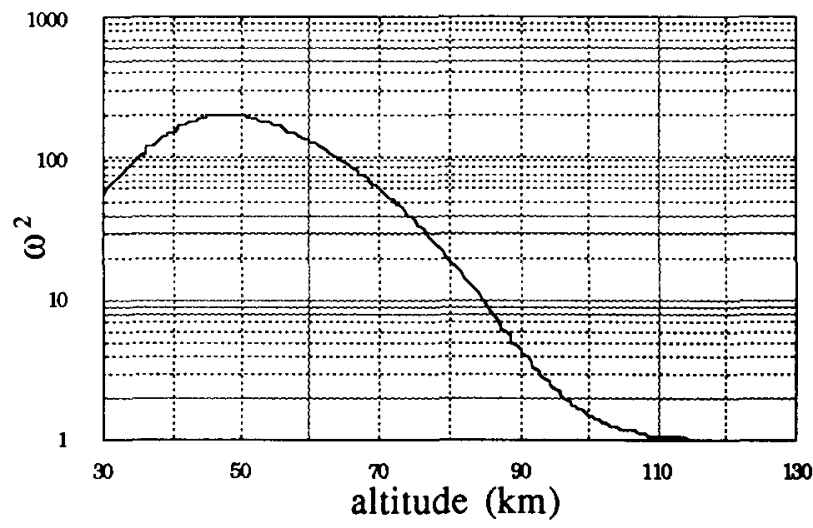

Fig. 3 Variation of $\omega^{2}$ with altitude for Etkin's vehicle 


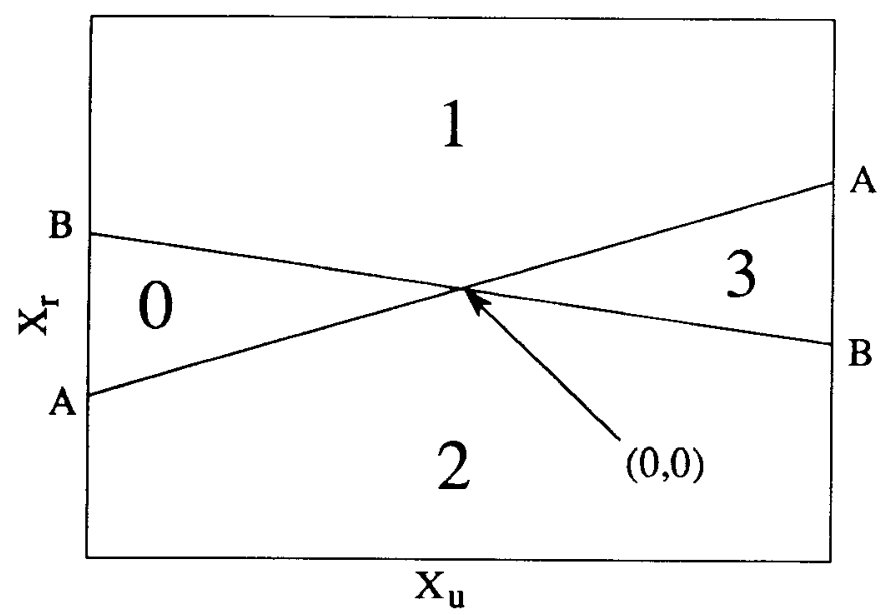

Fig. 4 Number of eigenvalues in the right-half plane as a function of the location on the plane of engine possibilities

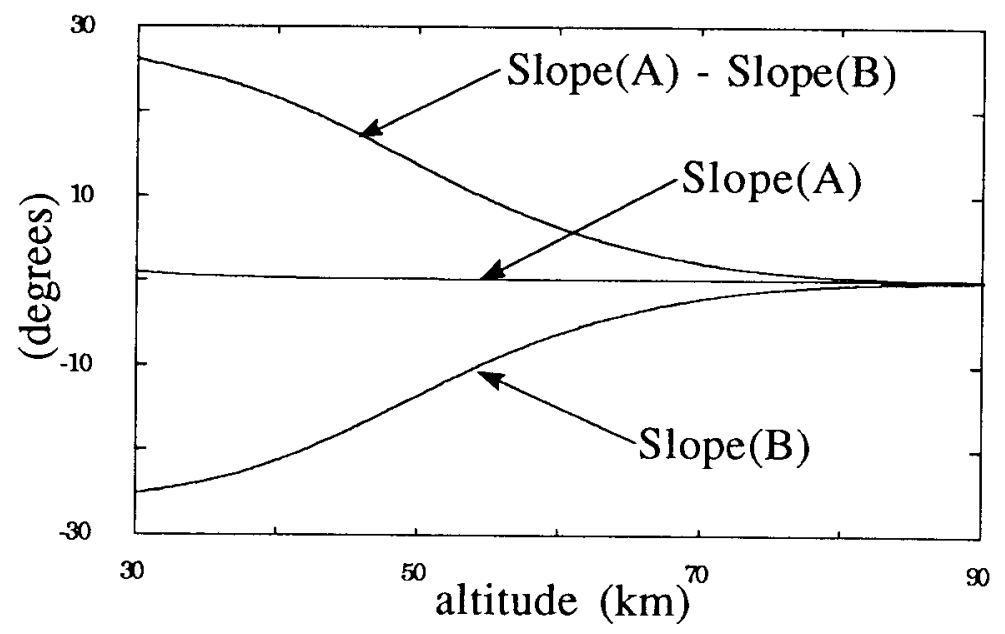

Fig. 5 Variation of the slopes of lines A and B with altitude for Etkin's vehicle

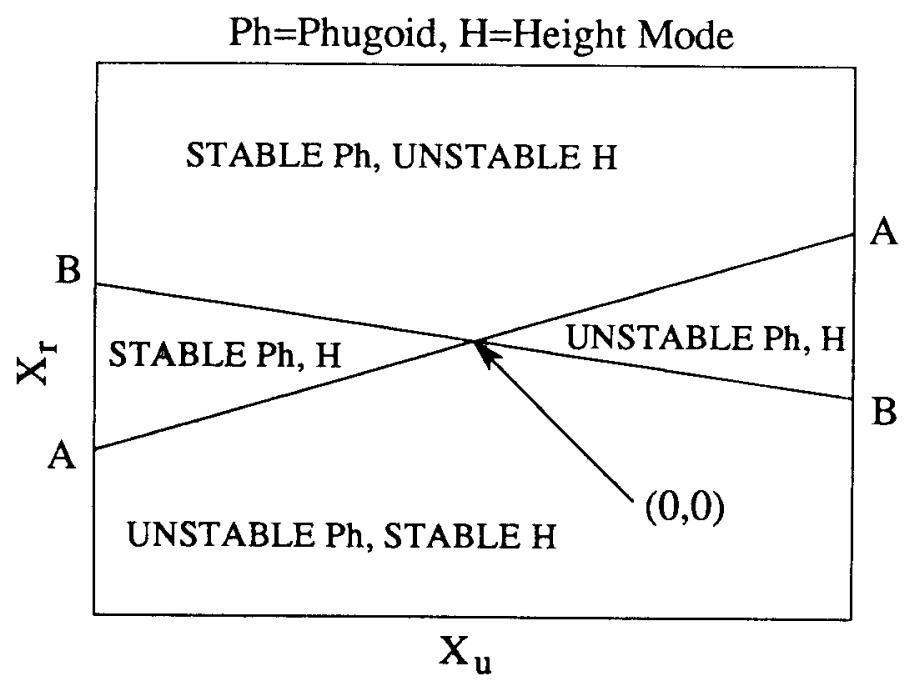

Fig. 6 General qualitative stability picture on the plane of engine possibilities 


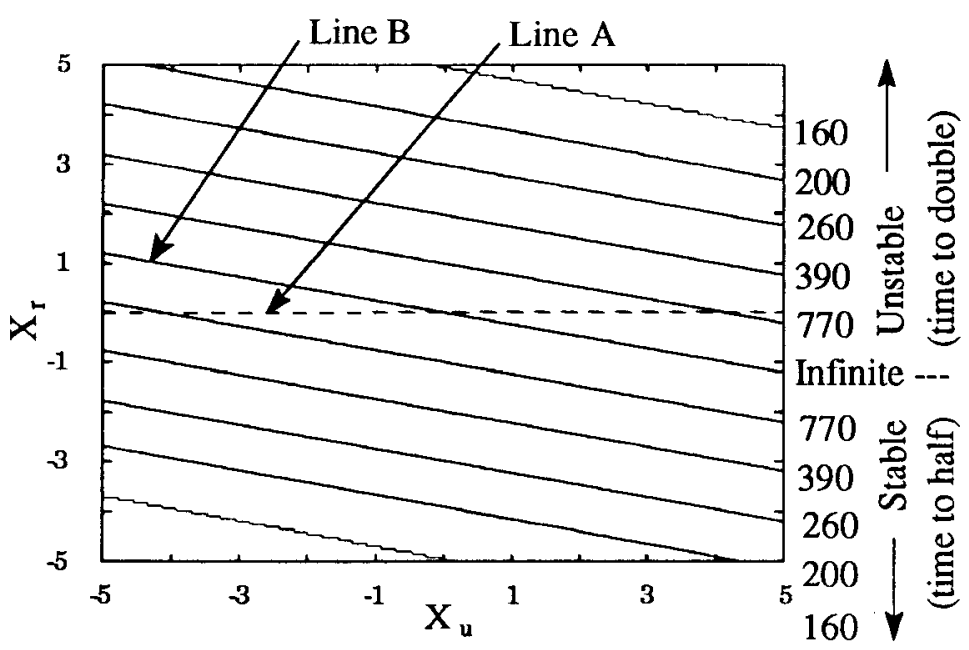

Fig. 7 Lines of constant height mode time to double/half (in sec) on the plane of engine possibilities for Etkin's vehicle at $50 \mathrm{~km}$

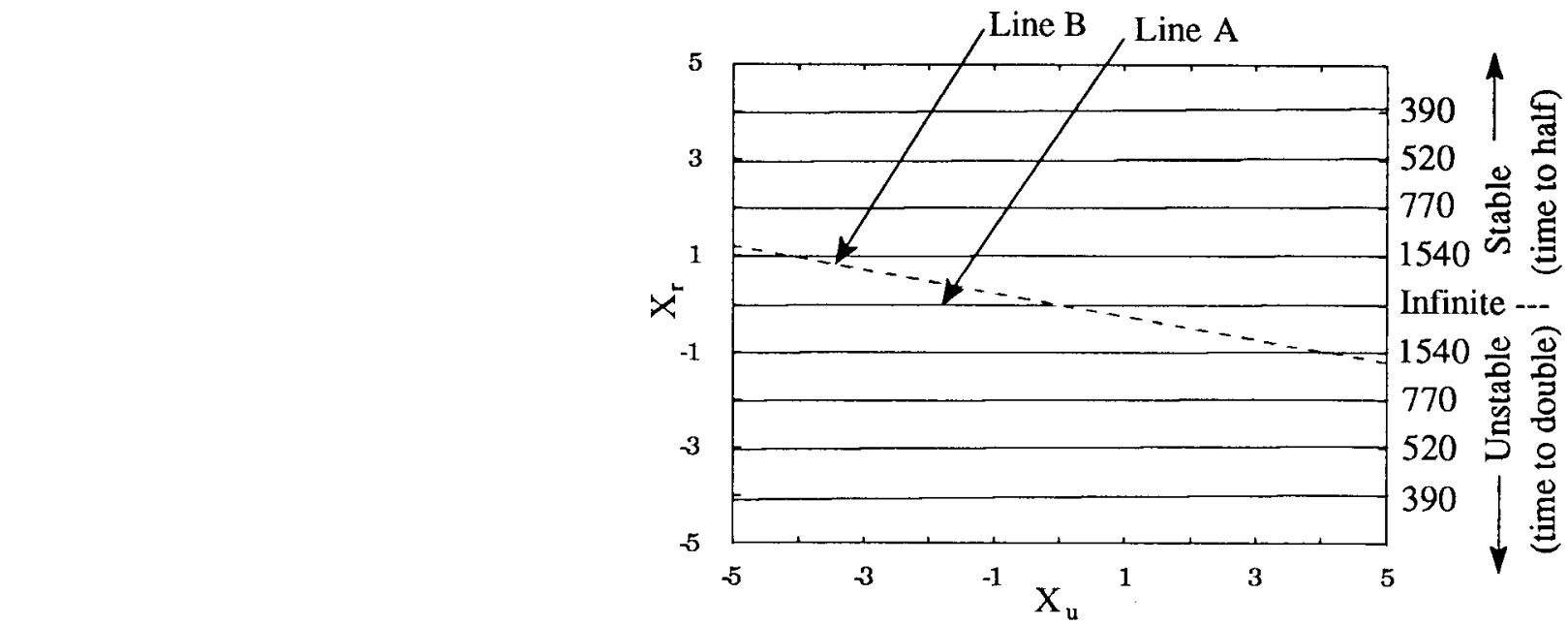

Fig. 8 Lines of constant phugoid amplitude time to double/half (in sec) on the plane of engine possibilities for Etkin's vehicle at $50 \mathrm{~km}$

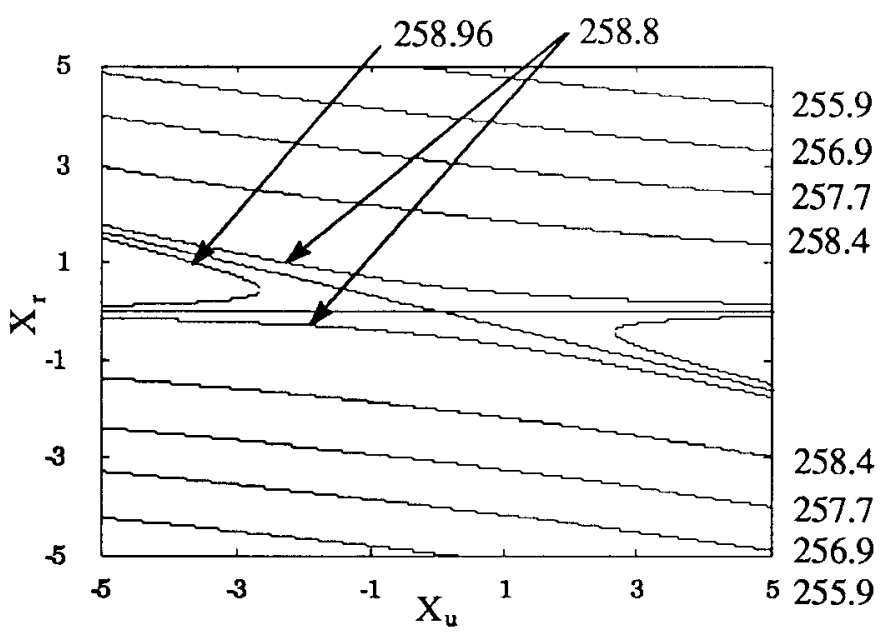

Fig. 9 Lines of constant phugoid period (in sec) on the plane of engine possibilities, for Etkin's vehicle at $50 \mathrm{~km}$ 


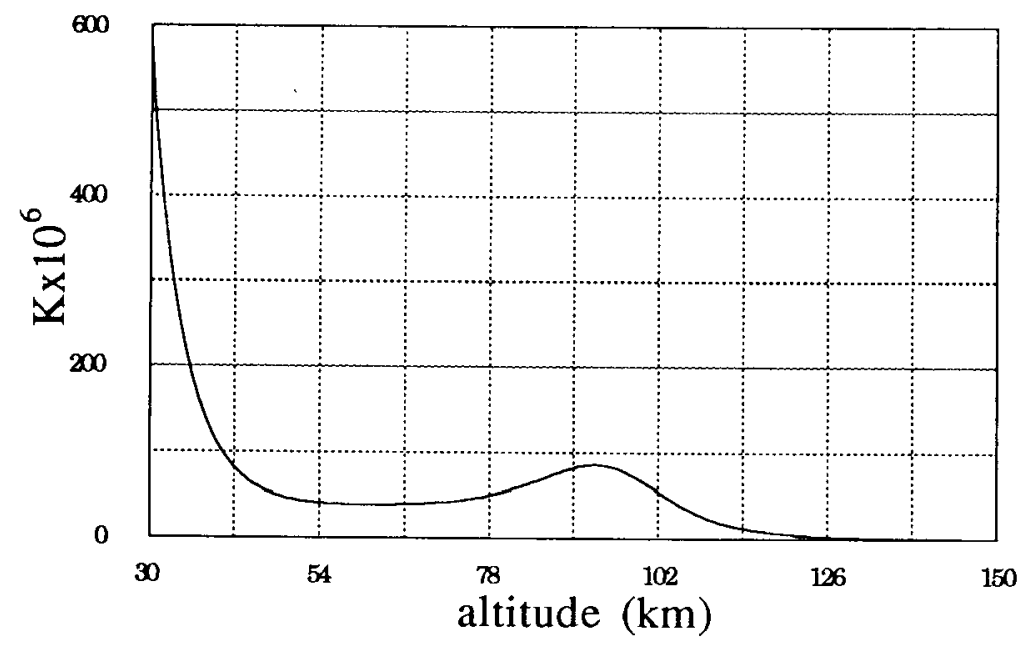

Fig. 10 Variation of $\mathrm{K}$ with altitude for Etkin's vehicle

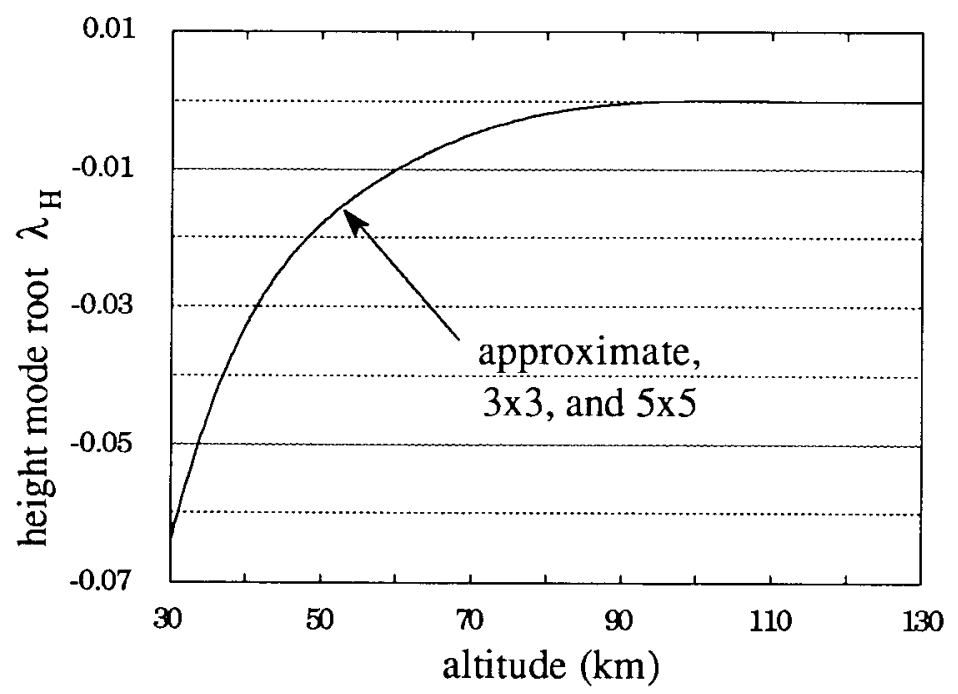

Fig. 11 Approximate (Eq. ( 59)), 3x3 (matrix AT T), and 5x5 (matrix A) results for the height mode for Etkin's vehicle, for a turbojet

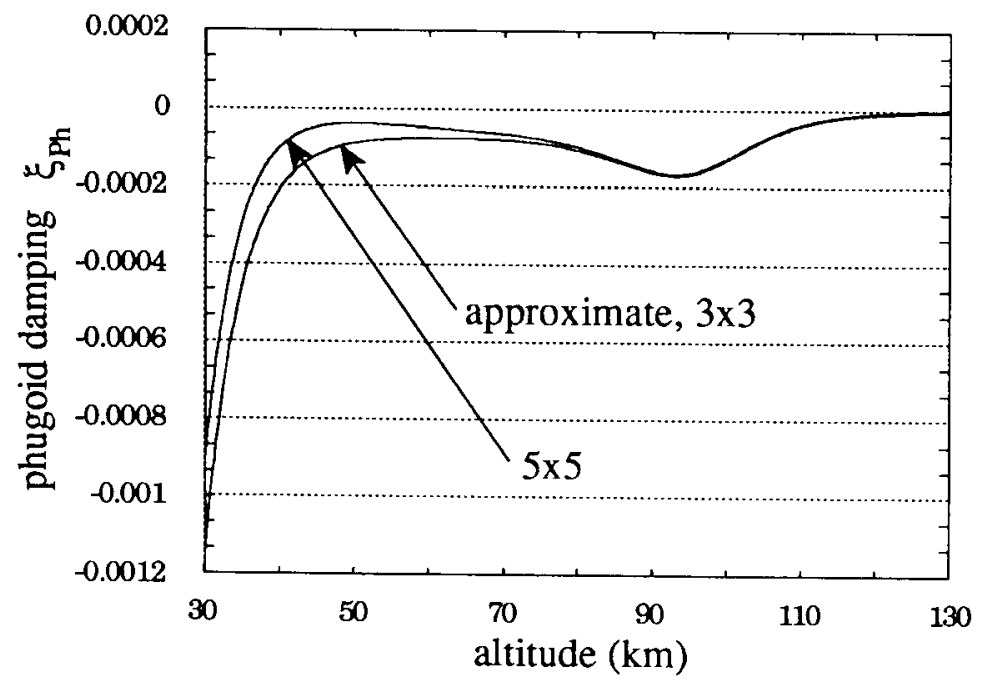

Fig. 12 Approximate (Eq. (60)), 3x3 (matrix AT T), and 5x5 (matrix A) results for the phugoid damping for Etkin's vehicle, for a turbojet 


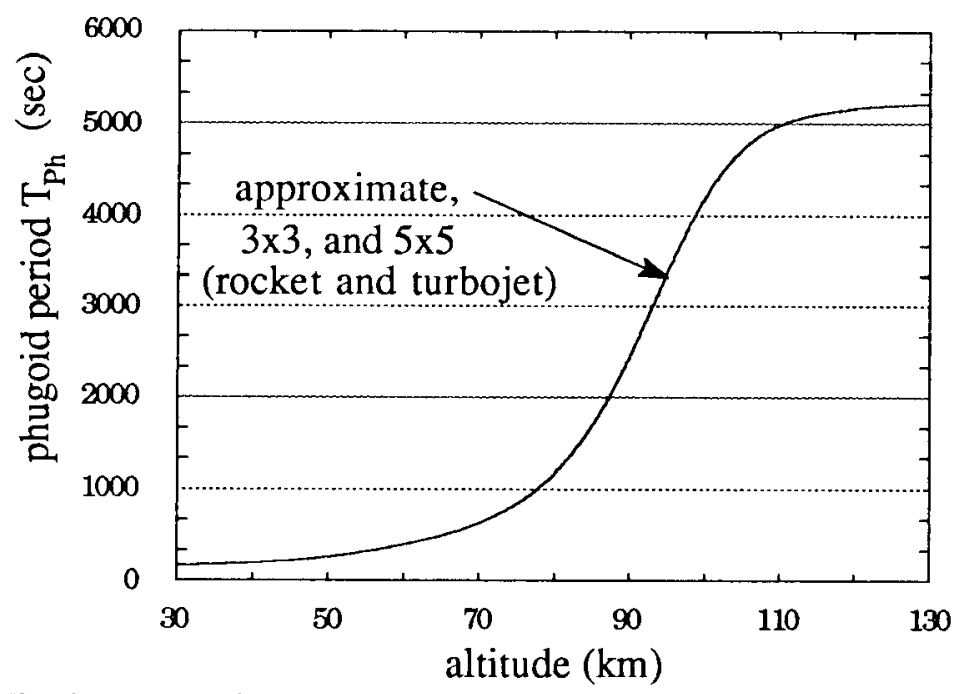

Fig. 13 Approximate (Eq. (65)), 3x3 (matrix AT T), and $5 \times 5$ (matrix A)

results for the phugoid period in real time for Etkin's vehicle

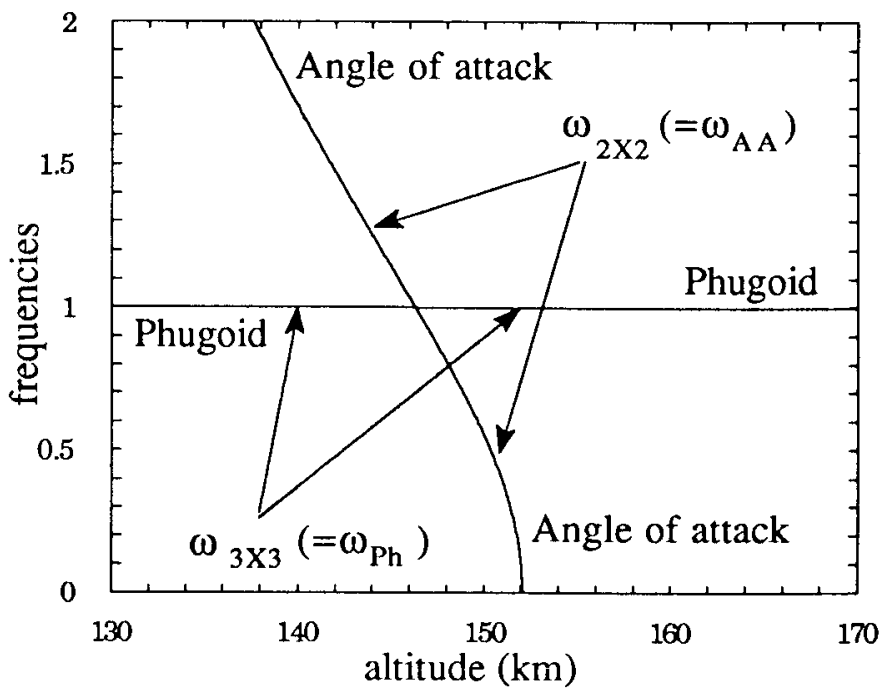

Fig. 14 Crossover of the $3 \times 3$ and $2 \times 2$ frequencies (associated with $A_{T} T$ and $A_{R} R$ respectively) at the resonance altitude $(\sim 146 \mathrm{~km})$ for Etkin's vehicle. The $5 \times 5$ frequencies have also been plotted and are

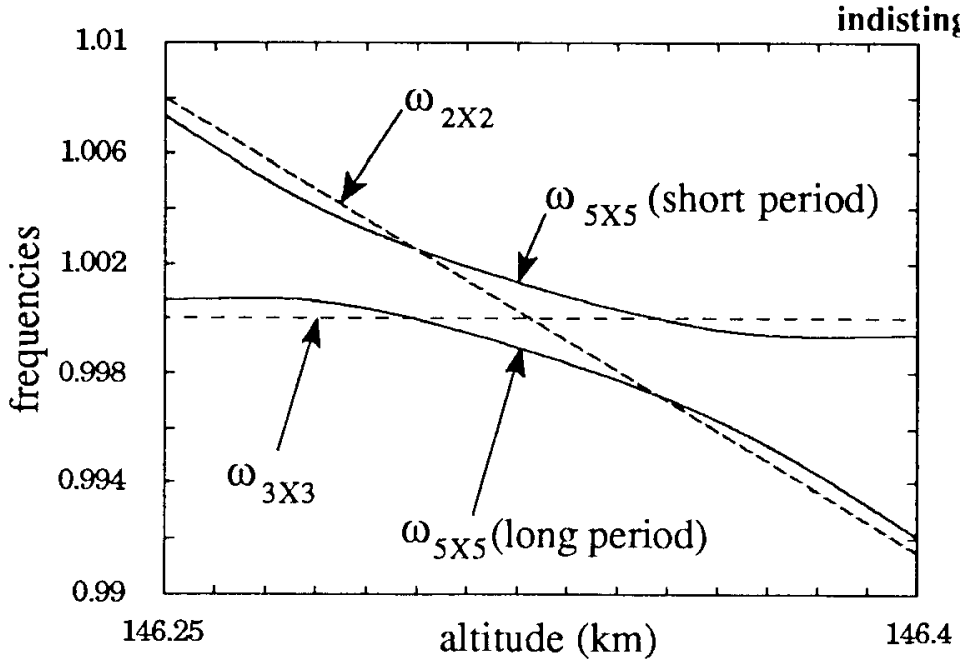

Fig. 15 Detail from Fig. 14, showing the situation at resonance altitude 